\title{
ESTABLECIMIENTO DE TRES ESPECIES ARBÓREAS EN LA CUENCA del ríO Magdalena, MÉxico
}

\author{
Silvia Castillo-Argüero', Yuriana Martínez-Orea y Guadalupe Barajas-Guzmán \\ Departamento de Ecología y Recursos Naturales, Facultad de Ciencias, Universidad Nacional Autónoma de México, \\ México D.F., México \\ 'autor para la correspondencia: silcas@ciencias.unam.mx
}

\begin{abstract}
Resumen: Se evaluó la supervivencia y crecimiento de tres especies arbóreas bajo diferentes tratamientos de micorrización $(Q u e r-$ cus rugosa), y cobertura vegetal (Abies religiosa y Pinus hartwegii) con el objetivo de recomendar los tratamientos para la restauración y manejo del bosque en la cuenca del río Magdalena. La supervivencia, la altura y el diámetro del tallo se evaluaron a lo largo de un año (agosto de 2006 a agosto de 2007). Los porcentajes de supervivencia más bajos se registraron en el tratamiento sin vegetación para A. religiosa y $P$. hartwegii. En el caso de $Q$. rugosa, en el tratamiento con micorrizas fue donde se presentó mayor supervivencia. Asimismo, las plantas de $Q$. rugosa con micorrizas alcanzaron significativamente un mayor crecimiento en altura que las no inoculadas. Los tratamientos utilizados fueron exitosos para las tres especies arbóreas, por lo que se recomienda reforestar adecuadamente con estas especies para un mejor manejo de cada tipo de vegetación dentro de la cuenca del río Magdalena.

Palabras clave: Abies religiosa, bosque templado, crecimiento, Pinus hartwegii, Quercus rugosa, supervivencia.
\end{abstract}

\begin{abstract}
Survival and growth of three tree species were evaluated under different treatments of mycorrhizal inoculation $(Q u e r-$ cus rugosa), and plant cover (Abies religiosa and Pinus hartwegii), with the aim to recommend treatments for restoration and forest management in the Magdalena river basin. Survival and stem height and diameter were measured for one year (August 2006 to August 2007). The lowest survival percentages were recorded for A. religiosa and P. hartwegii in the treatment without vegetation. The highest survival values for $Q$. rugosa corresponded to plants inoculated with mycorrhizae. Moreover, the inoculated $Q$. rugosa seedlings reached the highest height values when compared to those without mycorrhizae. The treatments proved to be successful for the three tree species; therefore, we recommend them for a reforestation plan in each vegetation type in the Magdalena river basin.
\end{abstract}

Keywords: Abies religiosa, growth, Pinus hartwegii, Quercus rugosa, survival, temperate forest.

$\mathbf{L}$ os bosques templados de la cuenca de México rodean uno de los mayores complejos urbanos del mundo, conocido como la zona Metropolitana de la Ciudad de México, la cual ha estado sujeta a perturbaciones antropogénicas desde tiempos precolombinos. A pesar de su importancia ecológica, esta zona enfrenta un continuo proceso de degradación de sus recursos naturales. El área contigua a la zona urbana es la que se encuentra más seriamente amenazada y que ha ido perdiendo, de manera constante, la capacidad de los ecosistemas para la recarga de acuíferos (Ávila-Akerberg y Almeida, 2009). Estos bosques se ven afectados por la tala ilegal, la incidencia de incendios, el cambio de uso de suelo y la contaminación de aire, agua y la falta de manejo técnico adecuado para su conservación (Manzanilla, 1974;
Rzedowski, 1978; Velázquez, 1994; Challenger, 1998; Alvarado-Rosales y Hernández-Tejeda, 2002).

A pesar de la experiencia que se tiene en el manejo silvícola y en la producción de algunas especies forestales, así como en el conocimiento acerca de la influencia de algunos factores ambientales en su desarrollo, no se ha resuelto de manera satisfactoria el problema de establecimiento exitoso de numerosas especies nativas características de estadíos sucesionales del bosque maduro; lo cual se debe, entre otras causas, a la falta de estudios en torno a su establecimiento y supervivencia, así como a la sustitución de las especies nativas por otras promovidas por los programas de reforestación (Nieto de Pascual-Pola, 2004; Montes-Merelles, 2006; Sandoval-González, 2010). 
México presenta una tasa elevada de deforestación, con un intervalo que va de 348,000 a 776,000 ha año-1 (Velázquez y Bocco, 2003). De éstas, aproximadamente se reforesta un 30\% (SEMARNAT, 2005); y de las plantas que se introducen, menos del 35\% sobreviven (Masera et al., 1997). Los bajos valores de supervivencia podrían deberse a la introducción de especies exóticas; además de la falta de seguimiento y cuidado de las plantas después del trasplante. De manera que la reforestación no ha tenido el éxito deseado para recuperar la cubierta forestal del territorio nacional (Cervantes et al., 2008). Además de que sería deseable aumentar el área reforestada, es necesario incrementar los porcentajes de supervivencia de las plantas utilizadas durante los planes de restauración. Dentro de las prácticas que podrían favorecer la supervivencia y el crecimiento de las plantas durante este proceso, se encuentran los tipos de cobertura vegetal que protegen la capa superficial del suelo, pues disminuyen la evaporación y, por tanto, el microclima es más favorable para el establecimiento de las plántulas trasplantadas (Maass y García-Oliva, 1990; Montaño y Monroy, 2000). Otra técnica es la inoculación de las raíces de las plantas con hongos para formar las micorrizas, mismas que al aumentar el área de absorción favorecen la eficiencia de captación de agua y nutrientes para las plantas (Brundrett et al., 1996), y con ello, aumentan la probabilidad de éxito del establecimiento de las plantas.

Este estudio evalúa la supervivencia y crecimiento de Abies religiosa, Pinus hartwegii y Quercus rugosa, especies arbóreas características de los bosques templados de la cuenca del río Magdalena (CRM), bajo diferentes tratamientos con el objetivo de poder recomendar las mejores combinaciones de desempeño para aplicarlas a la restauración y manejo del bosque en la CRM.

\section{Área de estudio}

La CRM es un área con una alta heterogeneidad ambiental y una gran riqueza específica (Ávila-Akerberg, 2004; Santibañez-Andrade, 2009). Actualmente, es una de las principales áreas de excedente hídrico de la ciudad de México (Mazari, 2000). Se han reportado 526 especies de plantas fanerógamas, pertenecientes a 92 familias y 274 géneros, lo que equivale a $2.3 \%$ del total de la estimación para la flora del país o al $25 \%$ de las plantas vasculares en la Cuenca de México (Ávila-Akerberg, 2004).

Los tipos de vegetación principales son los bosques de Pinus hartwegii Lindl. (Pino), Abies religiosa (HBK) Schlecht. (Oyamel) y Quercus rugosa Née-Quercus laurina Humb \& Bonpl. (Encinar), con distintas asociaciones de especies dentro de ellas (Figura 1; Ávila-Akerberg, 2002; Nava-López, 2003).

La CRM presenta microclimas que varían de templados a semifríos, según la clasificación climática de Köppen modificada por García (1978). Entre los 2,400 y 2,800 m de altitud se presenta el subtipo climático C (w2) (w) (b) i’g, templado subhúmedo con lluvias en verano, temperatura media anual entre 12 y $18^{\circ} \mathrm{C}$. En las partes más altas $(2,800$ a los 3,500 m) el clima es del tipo Cb' (w2) (w) (b') i g, semifrío, con temperaturas medias anuales entre 5 y $12{ }^{\circ} \mathrm{C}$. La precipitación aumenta con la altitud, con mínimos de 1,000 mm en las partes bajas y máximos de 1,500 $\mathrm{mm}$ en las cumbres más altas.

\section{CUENCA DEL RÍO MAGDALENA}

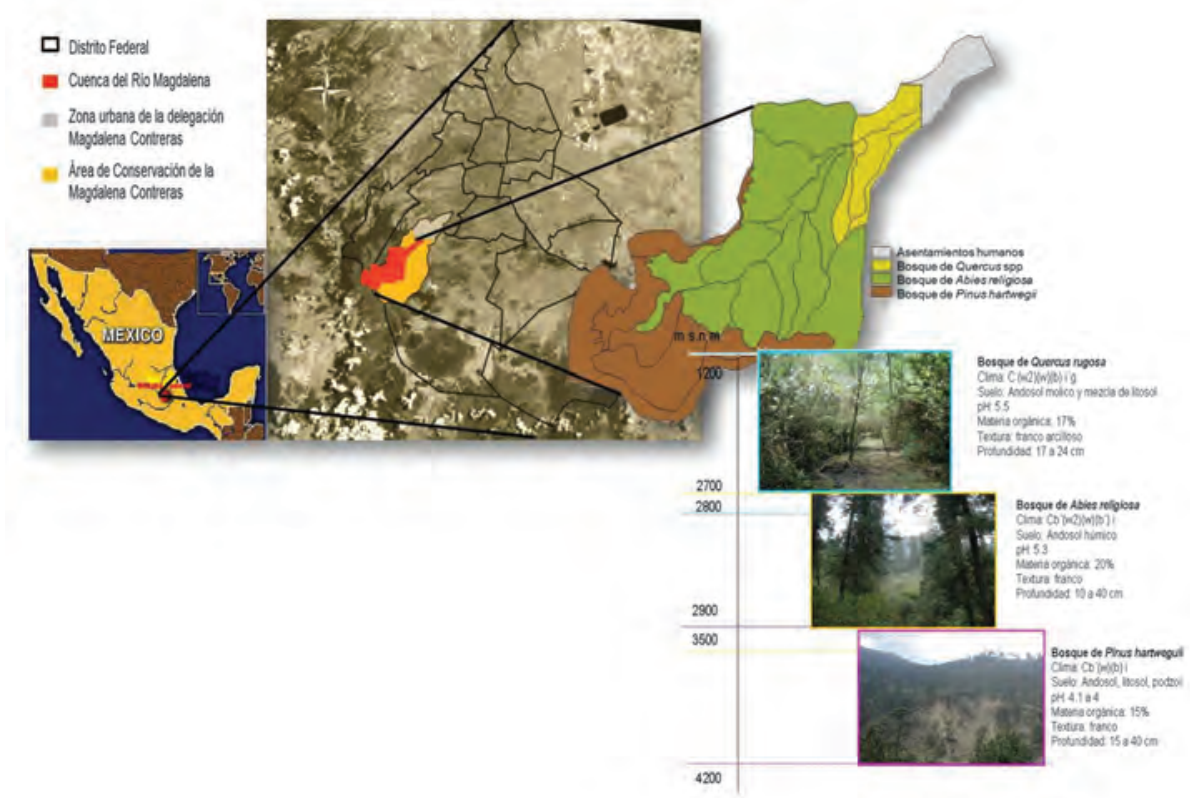

Figura 1. Mapa de ubicación de la cuenca del río Magdalena. 
Según la Clasificación Mundial de Suelos de la FAOUNESCO y adoptada por el INEGI (2013), predominan los andosoles, en su mayoría derivados de cenizas volcánicas. Son suelos muy ligeros, lo que condiciona una permeabilidad alta y un drenaje rápido y moderado; son ligeramente ácidos ( $\mathrm{pH}$ de 6.0 a 6.5) y muy heterogéneos en cuanto a su contenido de materia orgánica (CONAFOR, 2006). En algunas áreas, el andosol se combina con litosoles de textura media arenosa (COCODER, 1988; CONAFOR, 2006), estos últimos están representados en menor proporción y son suelos que presentan una profundidad menor a $10 \mathrm{~cm}$ y yacen sobre la roca. Se desarrollan en las pendientes mayores (12-20\%; CONAFOR, 2006). Los bosques de pino y oyamel se encuentran sobre andosoles y el paisaje está marcado por grandes claros, mientras que el bosque de encino se ubica sobre litosoles.

En la pasada última década se estima una pérdida anual de 240 ha de vegetación por cambio en el uso del suelo, que incluyen intereses inmobiliarios, la presencia de asentamientos irregulares, la atomización de parcelas agrícolas y los conflictos de tenencia de la tierra (CORENADER, 2003).

\section{Materiales y métodos}

En cada comunidad vegetal, durante un año (agosto de 2006 a agosto de 2007), se llevó a cabo el seguimiento mensual del crecimiento y supervivencia de plántulas de las tres especies arbóreas características del sistema. En cada una de éstas se establecieron parcelas permanentes, donde se sembraron las plántulas características de cada comunidad y, mensualmente, se midió el diámetro a la base del tallo (DAB) y la altura total (AT) de cada plántula trasplantada bajo los diferentes tratamientos. Las plántulas de Abies religiosa y de Pinus hartwegii se obtuvieron del vivero "Centro Forestal de San Luis Tlaxialtemalco". Las plántulas de Quercus rugosa se obtuvieron de semillas colectadas directamente en el campo.

Los tratamientos para las plantas de Quercus rugosa fueron con y sin micorrizas, ya que al encontrarse el bosque natural asentado en litosoles (Olivera-Morales et al., 2011), la poca profundidad del suelo y la textura gruesa que presentan, permiten el lavado del suelo y retardan el proceso de descomposición de la materia orgánica muerta, lo cual repercute en una baja cantidad de agua y nutrientes almacenados (Herrera, 2011), de manera que la micorriza podría beneficiar la absorción de agua y nutrientes, lo que favorece la supervivencia y crecimiento de las plantas (Olivera-Morales et al., 2011).

Para el caso de las plantas de Abies religiosa y Pinus hartwegii, los tratamientos fueron con vegetación cerrada y con vegetación abierta debido a la presencia natural de claros. En estas dos comunidades, la presencia de vegetación, como una cobertura viva, se ha reportado en la literatura como una ventaja para el establecimiento de estas especies, por lo que se decidió por estos tratamientos (González, 1985; Álvarez-
Cuadro 1. Descripción de los tratamientos que se llevaron a cabo para cada una de las tres especies que se introdujeron al campo.

\begin{tabular}{|c|c|c|c|}
\hline $\begin{array}{l}\text { Características de } \\
\text { los tratamientos }\end{array}$ & $\begin{array}{l}\text { Abies } \\
\text { religiosa }\end{array}$ & $\begin{array}{c}\text { Pinus } \\
\text { hartwegii }\end{array}$ & $\begin{array}{l}\text { Quercus } \\
\text { rugosa }\end{array}$ \\
\hline Número de parcelas & 2 & 2 & 1 \\
\hline Tamaños de parcelas (m) & ) $50 \times 50$ & $50 \times 50$ & $100 \times 100$ \\
\hline $\begin{array}{l}\text { Número de plántulas con } \\
\text { micorriza }\end{array}$ & n & - & 134 \\
\hline $\begin{array}{l}\text { Número de plántulas sin } \\
\text { micorriza }\end{array}$ & - & - & 134 \\
\hline $\begin{array}{l}\text { Número de plántulas en } \\
\text { vegetación cerrada }\end{array}$ & 120 & 200 & - \\
\hline $\begin{array}{l}\text { Número de plántulas en } \\
\text { vegetación abierta }\end{array}$ & 120 & 200 & - \\
\hline Forma de trasplante & $\begin{array}{c}\text { rodillo } \\
\text { alternada } \\
\text { cada } 2 \mathrm{~m}\end{array}$ & $\begin{array}{l}\text { rodillo } \\
\text { alternada } \\
\text { cada } 2 \mathrm{~m}\end{array}$ & $\begin{array}{l}\text { rodillo } \\
\text { alternada } \\
\text { cada } 2 \mathrm{~m}\end{array}$ \\
\hline $\begin{array}{l}\text { Edad de las plántulas } \\
\text { trasplantadas al campo } \\
\text { (meses) }\end{array}$ & 8 & 8 & 8 \\
\hline $\begin{array}{l}\text { Obtención de } \\
\text { la plántula }\end{array}$ & $\begin{array}{l}\text { vivero "Centro } \\
\text { Forestal de } \\
\text { San Luis } \\
\text { Tlaxialtemalco" }\end{array}$ & $\begin{array}{l}\text { vivero "Centro } \\
\text { Forestal de } \\
\text { San Luis } \\
\text { Tlaxialtemalco" }\end{array}$ & $\begin{array}{l}\text { por semilla } \\
\text { colectada } \\
\text { en campo }\end{array}$ \\
\hline
\end{tabular}

Moctezuma et al., 2012). En el cuadro 1 se especifican los diferentes tratamientos por especie.

Para la medición de las variables microambientales: la humedad del suelo se determinó bimestralmente por diferencia de peso entre muestras colectadas directamente del campo y muestras que se pusieron a secar a $105{ }^{\circ} \mathrm{C}$ por $48 \mathrm{~h}$. La temperatura del suelo se registró con cuatro censores automáticos H01, HOBO Onset Corporation USA, en cada uno de los tipos de vegetación y, para la temperatura y humedad del aire se utilizaron HOBOS H08B0 (Onset Co. EEUU)

Análisis. Para comparar la proporción de supervivientes entre tratamiento se llevó a cabo una prueba de $\mathrm{Z}$ y, la curva del porcentaje de supervivientes a través del tiempo se ajustó a un modelo lineal $(y=a+b x)$, con el objeto de hacer una representación más sencilla para las tres especies. Se utilizó el programa Table Curve 2D ver. 3 para determinar el porcentaje promedio de la población que muere por día.

Se calculó la tasa relativa de crecimiento (TRC) para diámetro (DAB) y altura (A) para las plántulas de cada una de las especies, de acurdo a la formula según Hunt (1978):

$$
\mathrm{TRC}=\ln \mathrm{A}_{\mathrm{f}}-\ln \mathrm{A}_{\mathrm{i}} / \mathrm{t}_{\mathrm{f}}-\mathrm{t}_{\mathrm{i}}
$$

Donde: $\mathrm{A}_{\mathrm{f}}$ y $\mathrm{A}_{\mathrm{i}}$ son las alturas o diámetros finales e iniciales de las plantas, $\mathrm{t}_{\mathrm{f}}$ es el tiempo final y $\mathrm{t}_{\mathrm{i}}$ el tiempo inicial (en días).

Se realizaron pruebas de $t$ de student para comparar entre tratamientos para cada especie, con el paquete estadístico STATISTICA 8 (StatSoft Inc., 2007), a un nivel de confianza del $95 \%$. 


\section{Resultados}

Supervivencia. La supervivencia de Quercus rugosa fue alta para ambos tratamientos (con o sin HMA). Sin embargo, la supervivencia fue mayor en las plantas que fueron inoculadas con HMA. La proporción de supervivencia final

A

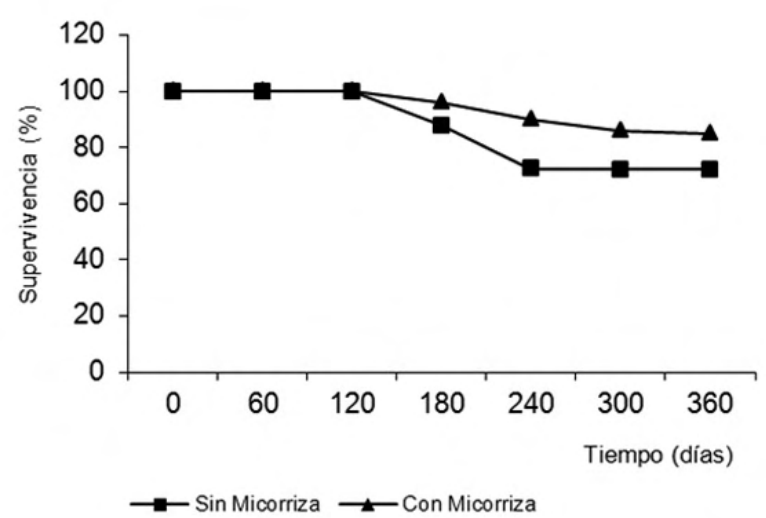

B

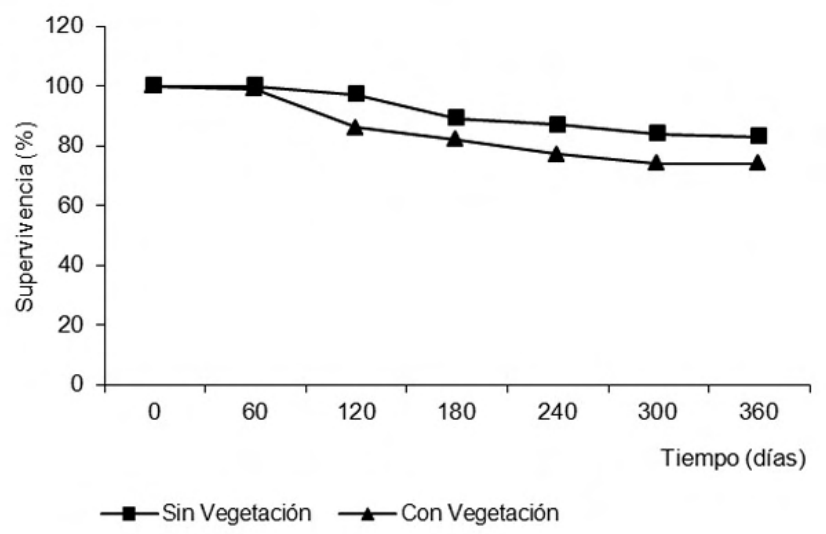

C

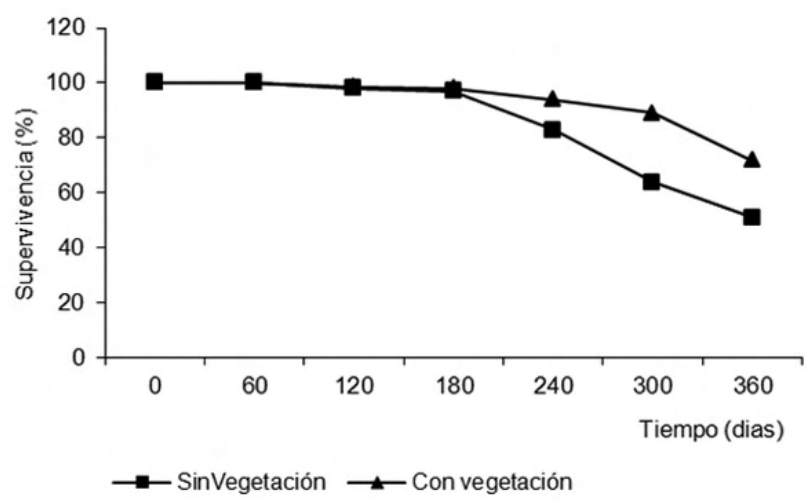

Figura 2. Porcentajes de supervivencia para A. Quercus rugosa, B. Abies religiosa y C. Pinus hartwegii en los diferentes tratamientos en el bosque templado de la cuenca del río Magdalena.
Cuadro 2. Valores de $r^{2}$ y tasas de mortalidad (b) para la supervivencia de Abies religiosa, Pinus hartwegii y Quercus rugosa durante un año, bajo diferentes tratamientos.

\begin{tabular}{lccc}
\hline & $\mathrm{r}^{2}$ & $\mathrm{~b}$ & $P$ \\
\hline Quercus rugosa & & & \\
Sin micorrizas & 0.85 & -0.1 & 0.0029 \\
Con micorrizas & 0.9 & -0.05 & 0.0011 \\
Abies religiosa & & & \\
Sin vegetación & 0.94 & -0.055 & 0.00035 \\
Con vegetación & 0.91 & -0.081 & 0.00083 \\
Pinus hartwegii & & & \\
Sin vegetación & & & \\
Con vegetación & 0.83 & -0.139 & 0.0045 \\
\hline
\end{tabular}

para plántulas no micorrizadas fue del $72 \%$, con 97 individuos, mientras que para las plántulas inoculadas la proporción fue del $84 \%$ con 113 supervivientes, la prueba de $\mathrm{Z}$ mostró diferencias significativas entre tratamientos $\left(\mathrm{Z}_{\mathrm{c}}=\right.$ $2.38, P<0.05)$.

La proporción de supervivencia de Abies religiosa fue del $81 \%$ en la zona sin vegetación (97 individuos), mientras que para la zona con vegetación sobrevivieron 88 individuos que corresponden a una proporción del $73 \%$. La prueba de $\mathrm{Z}$ demostró que no hay diferencias significativas en la proporción de supervivientes entre tratamientos $\left(\mathrm{Z}_{\mathrm{c}}=1.38, P>\right.$ 0.05). La mayor supervivencia en el caso de Pinus hartwegii correspondió a la zona con vegetación $(\mathrm{V}+)$, con una proporción de $72 \%$ que corresponde a 144 individuos; en tanto que la zona sin vegetación ( $\mathrm{V}$-) presento una proporción de $51 \%$, con 102 individuos vivos al final del experimento (Figura 2). La prueba de $\mathrm{Z}$ mostró diferencias significativas en el caso de la supervivencia $\left(Z_{c}=4.31, P<0.05\right)$.

El cuadro 2 muestra la velocidad promedio del porcentaje de la población para cada una de las especies que muere por día (tasa de mortalidad), las cuales se ajustaron al modelo lineal $y=\mathrm{a}+\mathrm{b} x$. La tasa de mortalidad de Quercus rugosa fue dos veces mayor para el tratamiento sin micorriza (M-) que para las que fueron inoculadas $(\mathrm{M}+)$. En el caso de Abies religiosa esta fue 1.4 veces mayor para las plántulas bajo el tratamiento con vegetación ( $\mathrm{V}+)$, en comparación de aquellas que fueron plantadas en zona sin vegetación ( $\mathrm{V}-$ ). Finalmente, Pinus hartwegii mostró que su tasa de mortalidad fue de 2.1 veces mayor para las plántulas sembradas en condiciones sin vegetación (V-) que las que se sembraron con vegetación $(\mathrm{V}+)$.

Crecimiento: La tasa relativa de crecimiento en Quercus rugosa mostró que los valores promedio de altura y $\mathrm{DAB}$ fueron ligeramente mayores en presencia de HMA, pero el análisis estadístico solo mostró diferencias significativas para la altura $\left(t_{0.05,208}=3.603, P<0.01\right)$. Donde la TRC en altura de las plantas inoculadas $(\mathrm{M}+)$ fue de $0.0011 \mathrm{~cm}$ 
$\mathrm{cm}^{-1}$ día $^{-1}$, y la de las plantas testigo (M-) fue de 0.0008 $\mathrm{cm} \mathrm{cm}^{-1}$ día $^{-1}$. La TRC del DAB fue la misma para ambos tratamientos $\left(0.0009 \mathrm{~cm} \mathrm{~cm}^{-1}\right.$ día $\left.^{-1}\right)$, de manera que no se presentaron diferencias significativas $\left(t_{0.05208}=0.32, P>\right.$ 0.05; Figura 3).

La TRC en altura y DAB en las plántulas de Abies religiosa que se establecieron en la zona con vegetación $(\mathrm{V}+)$ y en la zona sin vegetación (V-) no presentaron diferencias significativas entre tratamientos $\left(t_{0.05,183}=1.52, P>0.05 \mathrm{y}\right.$ $t_{0.05,183}=1.22, P>0.05$, respectivamente; Figura 3$)$. En el caso de Pinus hartwegii, la TRC en altura en las zonas con vegetación $(\mathrm{V}+)$ y sin vegetación ( $\mathrm{V}-$ ) no mostró diferencias significativas entre tratamientos $\left(t_{0.05,243}=0.73, P>0.05\right)$, y el DAB tampoco presentó diferencias $\left(t_{0.05,243}=0.87, P>\right.$ 0.05 ; Figura 3).

\section{Discusión}

El estadío de plántula es una etapa muy vulnerable dentro del ciclo de vida y muy importante durante el proceso de la regeneración. Las plántulas se involucran en relaciones inter e intraespecíficas al competir por los recursos que les permitirán crecer y establecerse entre las plantas de los diferentes estratos verticales del bosque, además de que el ambiente abiótico que las rodea puede llegar a ser estresante. Las respuestas de las plántulas ante este escenario se han asociado con el vigor propio de las plántulas en términos de la biomasa de la raíz y el diámetro del tallo, así como de la acción de la sombra y de las interacciones (Harcombe, 1987; Porter y Lowlor, 1991; Miquelajauregui-Graf, 2004).

Es importante tomar en cuenta que la tasa de crecimiento y la supervivencia se van a ver modificadas por la heterogeneidad biótica y abiótica intrínseca del sitio, así como por la generada por prácticas antrópicas (Harperet al., 1965; Salazar et al., 1999; Dominguez-Lerena et al., 2001). Esta heterogeneidad determina que las plántulas sobrevivan mejor en ciertos micrositios que en otros, y que el crecimiento también sea diferencial, de tal manera que se da una cierta "selección de hábitat", donde difiere la disponibilidad de recursos, principalmente de luz, nutrientes y agua (Kozlowski, 1999; Marino, 2004; Miquelajauregui-Graf, 2004), asociado a los atributos morfológicos y fisiológicos de las especies (Pacala et al., 1996; Kobe y Coates, 1997; LePage et al., 2000). Los porcentajes de supervivencia encontrados fueron contrastantes entre especies, el más bajo se presentó en las plántulas de Pinus hartwegii en el tratamiento sin vegetación (51\%) y el más alto en las plántulas de Quercus rugosa en el tratamiento con micorrizas (84\%). En el bosque de $P$. hartwegii se registraron los valores más bajos de temperatura y valores más altos en la oscilación térmica (GutiérrezUribe, 2011; Baltazar-Ortega, 2011), por lo que si las plantas no están protegidas por la presencia de cobertura vegetal resienten más el microclima y aumenta la mortalidad, las bajas temperaturas y la calidad de luz tienen una influen-
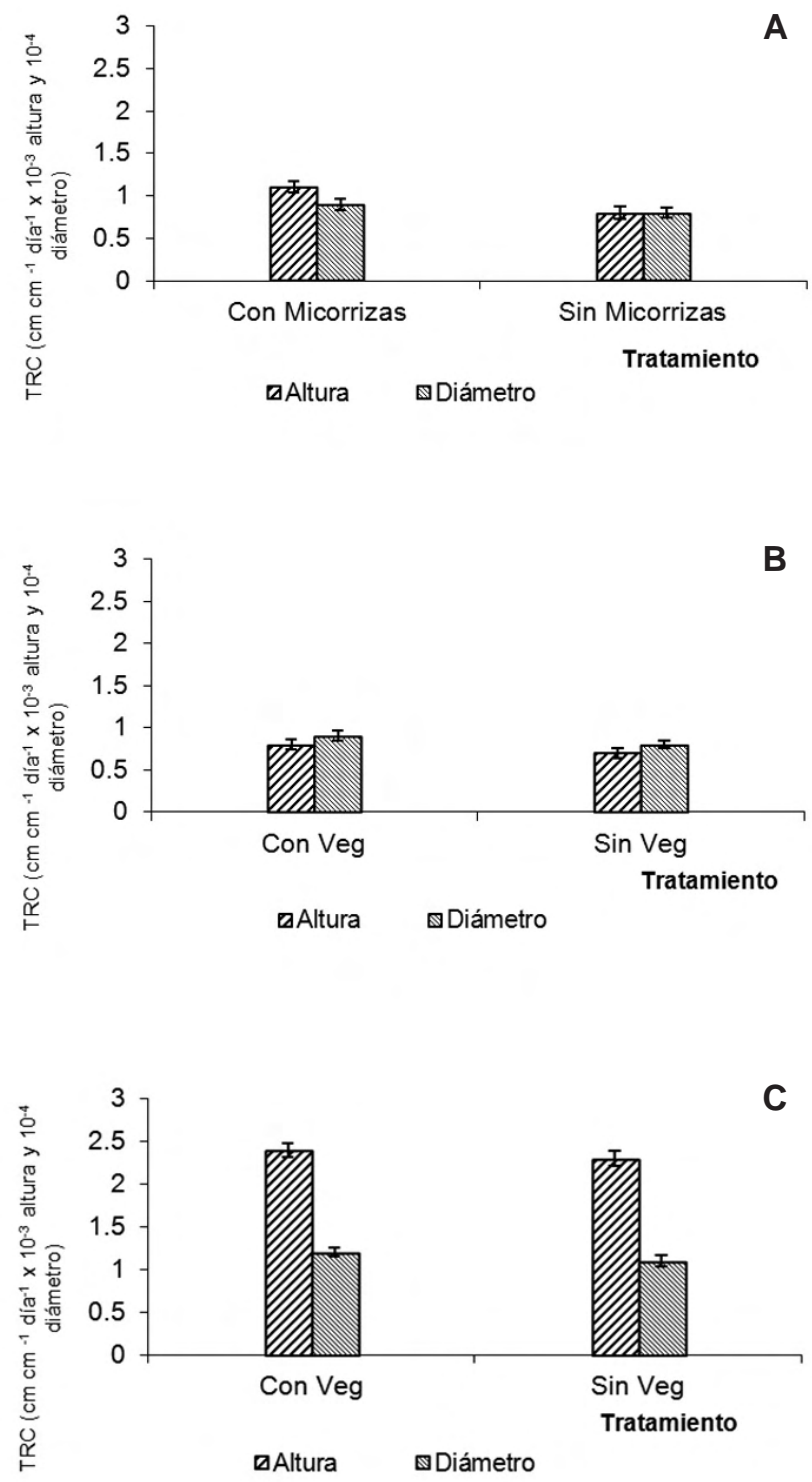

Figura 3. Tasa relativa de crecimiento (en altura y diámetro) de A. Quercus rugosa, B. Abies religiosa y C. Pinus hartwegii en los diferentes tratamientos en el bosque templado de la cuenca del río Magdalena.

cia en los resultados en crecimiento y supervivencia de las plantas a una edad temprana (DeLong et al., 1997; Paquette et al., 2006).

En el caso del bosque de Quercus se tiene el microclima más favorable, y a pesar de la poca profundidad del suelo, parece que las micorrizas desempeñaron un papel relevante en el alto porcentaje de supervivencia (Cuadro 3). Con relación a la regulación de las condiciones microclimáticas y el éxito en el establecimiento de las plántulas, Cabrelli et al. (2006) mencionan que las características microambientales modifican la cantidad y calidad de radiación solar que ingre- 
Cuadro 3. Características microclimáticas del aire y del suelo en los tres bosques en la Cuenca del Río Magdalena, D.F. Se presentan promedios \pm desviación estándar. Ta $=$ temperatura del aire, $\mathrm{Ts}=$ temperatura del suelo, $\mathrm{H}=$ humedad. Letras diferentes indican diferencias significativas entre los bosques $(P<0.05)$.

\begin{tabular}{lcrrrrr}
\hline Bosque & Ta media $\left({ }^{\circ} \mathrm{C}\right)$ & Ta mínima $\left({ }^{\circ} \mathrm{C}\right)$ & Ta máxima $\left({ }^{\circ} \mathrm{C}\right)$ & H aire $(\%)$ & Ts media $\left({ }^{\circ} \mathrm{C}\right)$ & $\mathrm{H} \mathrm{suelo}\left(\mathrm{g} \mathrm{g}{ }^{-1}\right)$ \\
\hline Pinus hartwegii & $6.8 \pm 0.2^{\mathrm{b}}$ & $-0.3 \pm 0.5^{\mathrm{c}}$ & $20.6 \pm 2.4^{\mathrm{a}}$ & $60 \pm 2^{\mathrm{b}}$ & $8.5 \pm 1.0^{\mathrm{ab}}$ & $0.41 \pm 0.9^{\mathrm{b}}$ \\
Abies religiosa & $6.9 \pm 0.1^{\mathrm{b}}$ & $2.9 \pm 0.3^{\mathrm{b}}$ & $13 \pm 0.3^{\mathrm{c}}$ & $65.4 \pm 8.7^{\mathrm{b}}$ & $7.0 \pm 1.3^{\mathrm{b}}$ & $0.82 \pm 0.3^{\mathrm{a}}$ \\
Quercus rugosa-Q. laurina & $11.2 \pm 0.03^{\mathrm{a}}$ & $6.9 \pm 0.03^{\mathrm{a}}$ & $16.9 \pm 0.2^{\mathrm{b}}$ & $75 \pm 0.07^{\mathrm{a}}$ & $9.9 \pm 0.5^{\mathrm{a}}$ & $0.65 \pm 0.2^{\mathrm{ab}}$ \\
\hline
\end{tabular}

sa en la cobertura, regulando así la temperatura y la humedad del aire y del suelo del micrositio. Asimismo, reportan que la presencia de cierta cobertura o de especies nodriza favorecen la supervivencia de coníferas. Al estar ubicadas cerca de una planta vecina (hierba o arbusto), las plántulas de $P$. hartwegii sobrevivieron aun en sitios abiertos durante la época invernal, favorecidas ante la existencia de un micrositio o "refugio" que evitó la desecación durante las heladas y periodos de congelamiento (Cabrelli et al., 2006; Sánchez-Gómez et al., 2006), lo que resulta vital si se considera que en la zona de estudio se han registrado temperaturas de $-3.64{ }^{\circ} \mathrm{C}$ de noviembre a febrero (Ramírez-Contreras y Rodríguez-Trejo, 2009; Blanco-García et al., 2011; Gutiérrez-Uribe, 2011).

Las plántulas de Abies religiosa sobrevivieron más en los sitios sin vegetación, aunque no se presentaron diferencias significativas con los sitios con vegetación. A las especies de este género se les relacionan tanto a los ambientes umbrófilos como heliófilos (Sánchez-Velásquez et al., 1991; Nieto de Pascual-Pola et al., 2003; Lara-González et al., 2009; Zetina-Galván, 2010). Sánchez-Velázquez et al. (1991) y Figueroa y Moreno (1993) mencionan que A. religiosa presenta mayor supervivencia en zonas arboladas, siempre y cuando existan los suficientes requerimientos de luz. En este trabajo, la tendencia a tener mayor supervivencia en ausencia de vegetación y de sombra podría confirmar la preferencia heliófila de la especie durante sus primeras etapas de desarrollo, como sucede con la mayoría de las coníferas (Lara-González et al., 2009; Zetina-Galván, 2010). Gordon (1970) concluyó que A. religiosa y A. magnifica tienen un bajo porcentaje de establecimiento en condiciones de luminosidad intensa debido a un ascenso en la temperatura del suelo; además, Nieto de Pascual-Pola (2004) menciona que A. religiosa sobrevive más en condiciones de 35 a $70 \%$ de sombra. Los resultados anteriores son parecidos a los que se obtuvieron, donde se observó que la supervivencia registrada en la zona sin vegetación fue ligeramente mayor a la observada en la zona con vegetación.

Para el caso de Quercus rugosa se debe considerar que, además de la presencia de los HMA, la supervivencia puede verse favorecida por la presencia de otras plantas que pudieron haber actuado como nodrizas para las plántulas de encino introducidas o para los propios HMA. Estas nodrizas en forma de árboles, arbustos y pastos, influyen en las condiciones microclimáticas de la zona de estudio, disminuyendo la exposición a los rayos del sol, lo que produce una menor temperatura y mayor disponibilidad de agua para las plantas (Gómez-Aparicio et al., 2005; Castro et al., 2006). Bonfil (1998) asegura que la presencia de nodrizas es altamente importante y aumenta las posibilidades de supervivencia de las plántulas de encino. En este sentido, al tener una mayor tasa de supervivencia de las plántulas inoculadas, la presencia de HMA es determinante para aumentar el establecimiento, ya que ayudan a la planta en situaciones de estrés, como la sequía (Rillig et al., 2002; Ramos-Zapata y Guadarrama, 2004).

En las zonas templadas, la mayoría de las especies leñosas presentan crecimiento en respuesta a la estacionalidad (Kozlowski et al., 1991; Salazar et al., 1999; Viveros-Viveros y Vargas-Hernández, 2007). En el periodo de baja temperatura y congelamiento, las plántulas entran en una fase de latencia, la cual consiste en que el meristemo apical y los meristemos laterales detienen su crecimiento. En las plántulas de Abies religiosa y de Pinus hartwegii este periodo de latencia se presentó entre los meses de enero a abril (aprox. 120 días), coincidiendo con la temporada más fría de la zona. Estas condiciones extremas de temperatura ocasionaron la ruptura del meristemo en algunos individuos, afectando su crecimiento en altura. Posiblemente por esta razón no se presentaron diferencias en ambas especies entre tratamientos. Al respecto, Repo (1992) reportó que durante la temporada de heladas, $P$. sylvestris fue más propenso a la ruptura del meristemo que Picea abies, a pesar de que ambas especies son resistentes al frío. En la zona de estudio, la temperatura promedio registrada durante este periodo fue de $-0.53{ }^{\circ} \mathrm{C}$ (Cuadro 3), observando en el suelo presencia de hielo. Sin embargo, los efectos del periodo invernal dependerán también de la edad del individuo y su ubicación (Kozlowski et al., 1991; Viveros-Viveros y Vargas-Hernández, 2007).

\section{Conclusiones}

Es importante hacer énfasis en que las tres especies estudiadas en este trabajo son potenciales para su uso en un programa de restauración de estos bosques. Los resultados muestran que la micorrización en las plántulas de Quercus resulta adecuada para aumentar la supervivencia de los in- 
dividuos trasplantados, de manera que se recomienda esta técnica para la recuperación del bosque de Quercus spp. en la CRM. Por otro lado, para la recuperación de las zonas deforestadas del bosque de Pinus hartwegii, se recomienda realizar el trasplante bajo árboles remanentes o en zonas que contengan vegetación herbácea, y en el caso del bosque de Abies religiosa resulta satisfactorio saber que el trasplante puede hacerse en zonas abiertas o cerradas y que el porcentaje de supervivencia estará por arriba de $70 \%$.

Se considera que es sumamente importante determinar las diferentes estrategias para restaurar o reforestar el bosque templado de la cuenca del río Magdalena, ya que es una zona proveedora de servicios ecosistémicos a la ciudad de México. Además de las herramientas propuestas en este trabajo se debe incluir un análisis del régimen de perturbación de la comunidad y la condición de fragmentación o cambio de uso de suelo en la que se encuentra el bosque a restaurar o reforestar.

\section{Agradecimientos}

Los autores, agradecemos a los revisores anónimos quienes mejoraron sustancialmente el documento, a Marco Antonio Romero-Romero por su apoyo en la elaboración de figuras. $\mathrm{Al}$ proyecto PAPIIT IN202210. A Mariana Gutiérrez, Jorge Zetina y Diego Olivera por su apoyo en el trabajo de campo. A la comunidad de la Magdalena Atlitic por el préstamo de las parcelas en los tres tipos de vegetación.

\section{Literatura citada}

Alvarado-Rosales D. y Hernández-Tejeda T. 2002. Decline of sacred fir in the Desierto de los Leones National Park. En: Fenn M.E., de Bauer L.I. y Hernandez-Tejeda T. Ed. Urban Air Pollution and Forests. Resources at Risk in the Mexico City Air Basin, Ecological Studies 156, pp. 243-260, Springer- Verlag, NuevaYork.

Álvarez-Moctezuma J.G., Ortiz-Reyes A.D, Sahagún-Castellanos J. y Peña-Lomelí A. 2012. Perfiles de rapd asociados con la resistencia a la intensidad luminosa alta en brinzales. Revista Mexicana de Ciencias Forestales 3:71-86.

Ávila-Akerberg V.D. 2002. La vegetación de la Cuenca Alta del Río Magdalena: Un enfoque florístico, fitosociológico y estructural. Tesis de licenciatura. Facultad de Ciencias, Universidad Nacional Autónoma de México. México, D.F. 86 pp.

Ávila-Akerberg V.D. 2004. Autenticidad de los bosques en la Cuenca del río Magdalena: Diagnóstico hacia la restauración. Tesis de Maestría. Facultad de Ciencias, Universidad Nacional Autónoma de México. México, D.F. 112 pp.

Ávila-Akerberg V.D. y Almeida L.L. 2009. Autenticidad forestal en el suroeste de la Ciudad de México. Hacia la restauración ecológica de los servicios ambientales. Revista Forestal Latinoamericana 24:31-53.

Baltazar-Ortega E.E. 2011. Variación espacio-temporal de la meso y macrofauna del mantillo en la cuenca alta del río Magdalena. Tesis de Licenciatura. Facultad de Ciencias, Universidad Nacional Autónoma de México. México, D.F. 70 pp.

Blanco-García A., Sáenz-Romero C., Martorell C., Alvarado-Sosa
P. y Lindig-Cisneros R. 2011. Nurse-plant and mulching effects on three conifer species in a Mexican temperate forest. Ecological Engineering 37:994-998.

Bonfil C. 1998. The effects of seed size, cotyledon reserves, and herbivory on seedlings survival and growth in Quercus rugosa and Q. laurina (Fagaceae). American Journal of Botany 85:79-87.

Brundrett M., Bougher N., Dell B., Grove T. y Malajczuk N. 1996. Working with mycorrhizas in forestry and agriculture. Austral Centre for International Agricultural. Monograph, Canberra.

Cabrelli D., Rebottaro S. y Effron D. 2006. Caracterización del dosel forestal y del microambiente lumínico en rodales con diferente manejo, utilizando fotografía hemisférica. Quebracho 13:17-25.

Castro J., Zamora R. y Hódar J.A. 2006. Restoring Quercus pyrenaica forests using pioneer shrubs as nurse plants. Applied Vegetation Science 9:137-142.

Cervantes G.V., Carabias L.J. y Arriaga V. 2008. Evolución de las políticas públicas de restauración ambiental en México. En: Carabias J., Mohar A., Anta S. y de la Maza J. Comps. Capital Natural de México. Vol III. Políticas Públicas y Perspectivas de Sustentabilidad, pp. 155-226, Comisión Nacional para el Conocimiento y uso de la Biodiversidad, México D.F.

Challenger A. 1998. Utilización y Conservación de los Ecosistemas Terrestres de México. Pasado, Presente y Futuro. Comisión Nacional para el Conocimiento y Uso de la Biodiversidad, Universidad Nacional Autónoma de México, México, D.F.

COCODER. Comisión Coordinadora para el Desarrollo Rural. 1988. Estudio de suelos de parte de la comunidad Magdalena Contreras. Delegación Magdalena Contreras. Comisión Coordinadora para el Desarrollo Rural: Subdirección Regional No. 1. México, D.F.

CONAFOR. Comisión Nacional Forestal. 2006. Programa Estratégico Forestal del Distrito Federal (PEF-DF) 2006 - 2025. Gobierno del Distrito Federal. Secretaria del Medio Ambiente. Dirección General de la Comisión de Recursos Naturales y Desarrollo Rural. México, D.F.

CONAFOR. Comisión Nacional Forestal. 2005. Programa Forestal y de Suelos, México. www.cnf.gob.mx:8080/snif/portal/

CORENADER. Conservación y Restauración de Recursos Naturales y Desarrollo Rural. 2003. El suelo de conservación del Distrito Federal. Secretaria de Medio Ambiente del Distrito Federal. México, D.F.

DeLong H.B., Lieffers V.J. y Bleins P.V. 1997. Microsite effects on first-year establishment and overwinter survival of white spruce in aspen-dominated boreal mixedwoods. Canadian Journal of Forest Research 27:1452-1457.

Dominguez-Lerena S., Villar S.P., Fuertes L. y Peñuleas R.J.L. 2001. ¿Puede la profundidad de plantación afectar la calidad fisiológica y al desarrollo en campo de los brinzales de Pinus halapensis? Actas del III Congreso Forestal Español 3:49-54.

Figueroa R. y Moreno M.S. 1993. Estructura y sucesión en poblaciones de Abies religiosa y Abies religiosa var. emarginata en la Sierra de Manantlán, Jalisco. Agrociencia 3:49-63.

García E. 1978. Los Climas del Valle de México. Colegio de Postgraduados, Secretaria de Agricultura y Recursos Hidráulicos. Chapingo.

Gómez-Aparicio L., Valladares F., Zamora R. y Quero J.L. 2005. Response of tree seedlings to the abiotic heterogeneity generated by nurse shrubs: an experimental approach at different scales. Ecography 28:757-768. 
González G.M.J. 1985. Comportamiento de la germinación y crecimiento inicial de Abies religiosa (H.B.K.) Schltdl. et Cham. en diferentes aperturas de dosel, preparaciones de suelo y variantes de siembra en Zoquiapan, Méx. Tesis de Licenciatura. Universidad Autónoma Chapingo. Texcoco, 82 pp.

Gordon D.T. 1970. Natural regeneration of white and red fir influence of several factors. Forest Service, United States Department of Agriculture. Berkeley.

Gutiérrez-Uribe M. 2011. Establecimiento y supervivencia de Pinus hartwegii Lindl., en una zona perturbada de la cuenca alta del río Magdalena. Tesis de Licenciatura. Facultad de Ciencias. Universidad Nacional Autónoma de México, México, D.F. 56 pp.

Harcombe P.A. 1987. Tree life tables. BioScience 37:557-568.

Harper J.L., Williams J.T. y Sagar G.R. 1965.The behavior of seeds in soil: I. The heterogeneity of soil surfaces and its role in determining the establishment of plants from seed. Journal of Ecology 53:273-86.

Herrera J. 2011. Geografía de suelos regional: Sierra Madre Occidental. En: Krasilnikov P., Jiménez N.F.J., Reyna T.T. y García C.N.E. Eds. Geografía de Suelos de México. Las Prensas de Ciencias. Universidad Nacional Autónoma de México. México, D.F.

Hunt R. 1978. Plant Growth Analysis. E. Arnold, Londres.

INEGI, 2013. Prontuario de información geográfica delegacional de los Estados Unidos Mexicanos. La Magdalena Contreras, Distrito Federal Clave geoestadística 09008.

Kobe R.K. y Coates K.D. 1997. Models of sapling mortality as a function of growth to characterize interspecific variation in shade tolerance of eight tree species of northwestern British Columbia. Canadian Journal of Forest Research 27:227-236.

Kozlowski T.T., Kramer P.J. y Pallardy S.G. 1991. The Physiological Ecology of Woody Plants. Academic Press. Nueva York.

Kozlowski T.T. 1999. Soil compaction and growth of woody plants. Scandinavian Journal of Forest Research 14:596-619.

Lara-González R., Sánchez-Velásquez L.R. y Corral-Aguirre J. 2009. Regeneración de Abies religiosa en claros del dosel versus sotobosque, Parque Nacional Cofre de Perote, México. Agrociencia 43:739-747.

LePage P.T., Canham C.D., Coates K.D. y Bartemucci P. 2000. Seed abundance versus substrate limitation of seedling recruitment in northern temperate forests of British Columbia. Canadian Journal of Forest Research 30:415-427.

Maass J.M. y García-Oliva F. 1990. La investigación sobre erosión de suelos en México: un análisis de la literatura existente. Ciencia 41:209-228.

Manzanilla H. 1974. Investigaciones epidométricas y silvícolas en bosques mexicanos de Abies religiosa. Dirección General de Información y Relaciones Públicas de la Secretaría de Agricultura y Ganadería, México, D.F.

Marino C.H. 2004. Fisiología Ecológica en plantas. Mecanismos y Respuestas a Estrés en los Ecosistemas. Ediciones Universitarias de Valparaíso. Santiago de Chile.

Masera O., Bellon M.R. y Segura G. 1997. Forestry Options for Sequestering Carbon in Mexico: Comparative Economic Analysis of Three Case Studies. Critical Reviews in Environmental Science and Technology 27(suppl.):227-244.

Mazari M.M. 2000. Dualidad Población-Agua: Inicio del Tercer Milenio. El Colegio Naciona. México D.F.

Miquelajauregui-Graf Y. 2004. Estudio comparativo de la sobre- vivencia y el crecimiento temprano de dos especies de Neobuxbaumia (Cactaceae) que difieren en su nivel de rareza. Tesis de Licenciatura de Biología. Facultad de Ciencias. Universidad Nacional Autónoma de México. México, D.F. 78 pp.

Montaño A.N.M. y Monroy A.A. 2000. Conservación ecológica de suelos en zonas áridas y semiáridas de México. Ciencia y Desarrollo 154:27-37.

Montes-Merelles L.C. 2006. Crecimiento y supervivencia de plántulas de Bursera glabrifolia en respuesta a diferentes condiciones ambientales. Tesis de Licenciatura de Biología. Facultad de Ciencias. Universidad Nacional Autónoma de México, México, D.F. 71 pp.

Nava-López M.Z. 2003. Los bosques de la cuenca alta del río Magdalena, D.F., México. Un estudio de vegetación y fitodiversidad. Tesis de Licenciatura de Biología. Facultad de Ciencias. Universidad Nacional Autónoma de México, México, D.F. 64 pp.

Nieto de Pascual-Pola C., Musálem M.A. y Ortega-Alcalá J. 2003. Estudio de algunas características de conos y semillas de Abies religiosa (HBK) Schl. et Cham. Agrociencia 37:521-531.

Nieto de Pascual-Pola M.C.C. 2004. Regeneración del oyamel (Abies religiosa (HBK) Schltdl. et Cham.) bajo condiciones naturales y controladas. Tesis de Posgrado en Ciencias Biológicas, Facultad de Ciencias. Universidad Nacional Autónoma de México, México, D.F. 148 pp.

Olivera-Morales D., Castillo-Argüero S., Guadarrama P., RamosZapata J., Álvarez-Sánchez J. y Hernández-Cuevas L. 2011. Establecimiento de plántulas de Quercus rugosa Née inoculadas con hongos micorrizógenos arbusculares en un bosque templado de México. Boletín de la Sociedad Botánica de México 89:115-121.

Pacala S.W., Canham C.D., Saponara J., Silander J.A.Jr., Kobe R.K. y Ribbens E. 1996. Forest models defined by field measurements: estimation, error analysis, and dynamics. Ecological Monographs 66:1-44.

Paquette A., Bouchard A. y Cogliastro A. 2006. Survival and growth of under-planted trees: a meta-analysis across four biomes. Ecological Applications 16:1575-1589.

Porter J.R. y Lowlor D.W. 1991. Plant Growth Interactions with Nutrition and Environment. Cambridge University Press. Cambridge.

Ramírez-Contreras A., Rodríguez-Trejo D.A. 2009. Plantas nodriza en la reforestación con Pinus hartwegii Lindl. Revista Chapingo Serie Ciencias Forestales y del Ambiente 15:43-48.

Ramos-Zapata J. y Guadarrama P. 2004. Los hongos micorrizógenos arbusculares en la restauración de comunidades tropicales. Universidad y Ciencia 1(núm. Especial):59-65.

Repo T. 1992. Seasonal changes of frost hardiness in Picea abies and Pinus sylvestris in Finland. Canadian Journal of Forest Research 22:1949-1957.

Rillig M.C., Wright S.F., Shaw M.R. and Field C.B. 2002. Artificial climate warming positively affects arbuscular mycorrhizae buy decreases soil aggregate water stability in an annual grassland. Oikos 97:52-58.

Rzedowski J. 1978. Vegetación de México. Limusa, México D.F.

Salazar G.J.G., Vargas H.J.J., Jasso M.J., Molina G.J.D., Ramírez H.C. y López U.J. 1999. Variación en el patrón de crecimiento en altura de cuatro especies de Pinus en edades tempranas. $M a$ dera y Bosques 5:19-34.

Sánchez-Gómez D., Zavala M.A. y Valladares F. 2006. Seedling survival responses to irradiance are differentially influence by 
low-water availability in four tree species of the Iberian cool temperate-Mediterranean ecotone. Acta Oecologica 30:322-332.

Sánchez-Velásquez L.R., Pineda-López M.R. y Hernández-Martínez A. 1991. Distribución y estructura de la población de Abies religiosa (H.B.K.) Schlecht. et Cham., en el Cofre de Perote, estado de Veracruz, México. Acta Botanica Mexicana 16:45-55.

Sandoval-González I. 2010. Efecto de los hongos ectomicorrizógenos en el crecimiento y supervivencia de plántulas de Pinus hartwegii Lindl. y Abies religiosa (Kunth Schltdl. et Cham.): Un enfoque para la restauración de ambientes deteriorados en la Cuenca del Río Magdalena D.F. Tesis de Maestría en Ciencias Biológicas. Facultad de Ciencias. Universidad Nacional Autónoma de México. México, D.F. 99 pp.

Santibañez-Andrade G. 2009. Composición y estructura de bosque de Abies religiosa en función de la heterogeneidad ambiental y determinación de su grado de conservación en la cuenca del Río Magdalena, México., D.F. Tesis de Maestría en Ciencias Biológicas. Facultad de Ciencias. Universidad Nacional Autónoma de México. 134 pp.

SEMARNAT. Secretaría de Medio Ambiente y Recursos Natu- rales. 2005. Informe de la Situación del Medio Ambiente en México. Compendio de estadísticas ambientales. México, D.F.

StatSoft Inc. 2007. STATISTICA para Windows. Versión Manual 6.0. Tulsa. Oklahoma.

Velázquez A. 1994. Multivariate analysis of the vegetation of the volcanoes Tláloc and Pelado, Mexico. Journal of Vegetation Science 5:263-270.

Velázquez A. y Bocco G. 2003. La ecología del paisaje y su potencial para acciones de conservación de ecosistemas templados de montaña. En: Sánchez O., Vega E., Peters E. y Monroy-Vilchis O. Eds. Conservación de Ecosistemas Templados de Montaña en México, pp. 175-194, Instituto Nacional de Ecología, México, D.F.

Viveros-Viveros H. y Vargas-Hernández J.J. 2007. Dormancia en yemas de especies forestales. Revista Chapingo Serie Ciencias Forestales y del Ambiente 13:131-135.

Zetina-Galván J. 2010. Crecimiento y supervivencia de Abies religiosa (H.B.K) Schltdl. \& Cham. bajo diferentes tratamientos de cobertura vegetal, en San Nicolás Totolapan, D.F.. Tesis de Licenciatura. Facultad de Ciencias. Universidad Nacional Autónoma de México, México D.F. 66 pp.

Recibido: 4 de junio de 2013

Aceptado: 26 de julio de 2013 